\title{
Partial Semantics for Quantified Modal Logic
}

\author{
Eric Johannesson ${ }^{1}$ (D)
}

Received: 20 June 2017 / Accepted: 6 April 2018 / Published online: 8 May 2018

(C) The Author(s) 2018

\begin{abstract}
When it comes to Kripke-style semantics for quantified modal logic, there's a choice to be made concerning the interpretation of the quantifiers. The simple approach is to let quantifiers range over all possible objects, not just objects existing in the world of evaluation, and use a special predicate to make claims about existence (an existence predicate). This is the constant domain approach. The more complicated approach is to assign a domain of objects to each world. This is the varying domain approach. Assuming that all terms denote, the semantics of predication on the constant domain approach is obvious: either the denoted object has the denoted property in the world of evaluation, or it hasn't. On the varying domain approach, there's a third possibility: the object in question doesn't exist. Terms may denote objects not included in the domain of the world of evaluation. The question is whether an atomic formula then should be evaluated as true or false, or if its truth value should be undefined. This question, however, cannot be answered in isolation. The consequences of one's choice depends on the interpretation of molecular formulas. Should the negation of a formula whose truth value is undefined also be undefined? What about conjunction, universal quantification and necessitation? The main contribution of this paper is to identify two partial semantics for logical operators, a weak and a strong one, which uniquely satisfy a list of reasonable constraints (Theorem 2.1). I also show that, provided that the point of using varying domains is to be able to make certain true claims about existence without using any existence predicate, this result yields two possible partial semantics for quantified modal logic with varying domains.
\end{abstract}

Eric Johannesson

johannesson.eric@gmail.com

1 Filosofiska Institutionen, Stockholms Universitet, SE-106 91 Stockholm, Sweden 
Keywords Quantified modal logic · Varying domains · Semantics · Existence · Partial semantics

\section{Introduction}

When it comes to the semantics for quantified modal logic, there's a choice to be made concerning the interpretation of the quantifiers. The simple approach is to let quantifiers range over all possible objects, not just objects existing in the world of evaluation, and use a special predicate to make claims about existence (an existence predicate). This is the constant domain approach. The more complicated approach is to assign a domain of objects to each world. That is the varying domain approach.

Allegedly, the problem with the simple approach is that it makes for an unnatural interpretation of the quantifiers, allowing us to truthfully say things like

(1) There are things that don't exist.

which, on the face of it, seems contradictory. On the other hand, one might want to distinguish between there is and exists. To take an example borrowed from E. N. Zalta, it seems we can truthfully say things like

(2) There are literary characters that, although they don't exist, continue to inspire many readers.

Assuming that all terms denote, the semantics of predication on the constant domain approach is obvious: either the denoted object has the denoted property in the world of evaluation, or it hasn't. On the varying domain approach, there's a third possibility: the object in question doesn't exist. Terms may denote objects not included in the domain of the world of evaluation. The question is whether an atomic formula then should be evaluated as true or false, or if its truth value should be undefined. Leaving it undefined will have ramifications for the interpretation of various operators. Should the negation of a formula whose truth value is undefined also be undefined? What about conjunction, universal quantification and necessitation? The same questions arise for non-denoting terms (e.g. definite descriptions such as the present King of France). Unfortunately, it's common to lump these two phenomena together under the label empty terms, i.e. terms that either denote something outside the domain of quantification, or don't denote anything at all. ${ }^{1}$ I can see no obvious reason why these should be treated in a unified fashion, so I will not. For my purposes, I will assume that the truth value of an atomic formula containing a non-denoting term is undefined. Under that assumption, I will investigate what the value of an atomic formula containing terms denoting something outside the domain of the world of evaluation should be.

\section{Quantified Modal Logic}

We begin by introducing some standard notions. Let $\mathcal{L}_{\square}$ be a language of first order logic extended by a sentence-forming operator $\square$ (box), where the intended

\footnotetext{
${ }^{1}$ See, for instance, [4].
} 
interpretation of $\square \varphi$ is something like It's necessarily the case that $\varphi$. Furthermore, let $\mathcal{L}_{\square \iota}$ be $\mathcal{L}_{\square}$ extended by a term-forming quantifier $\iota$ (inverted iota), where the intended interpretation of $\iota \varphi$ is something like the object such that $\varphi$. So, for instance, the denotation of the present king of France shall be undefined. We shall take the operators $\neg, \wedge, \forall$ and $\square$ as primitive, and treat other operators such as $\vee, \exists$ and $\diamond$ as abbreviations in the usual way. We introduce the notion of a frame, and define the notion of a model or interpretation of $\mathcal{L}_{\square \iota}$ (and its fragment $\mathcal{L}_{\square}$ ) in terms of it:

Definition 2.1 (Frame) A frame is a quadruple $\mathcal{F}=\langle W, D, D o m, R\rangle$, where $W$ is a non-empty set (a set of possible worlds), $R$ is a binary relation on $W$ (an accessibility relation), $D$ is a non-empty set (a domain of possible objects) and Dom : $W \rightarrow \mathcal{P}(D)$ is a function assigning a set of objects to each world (the set of objects existing in that world). A constant frame is a frame $\mathcal{F}=\langle W, D, \operatorname{Dom}, R\rangle$ such that $\operatorname{Dom}(w)=$ $\operatorname{Dom}\left(w^{\prime}\right)=D$ for all $w, w^{\prime} \in W$.

Definition 2.2 (Model) A model of $\mathcal{L}_{\square \iota}$ is a tuple $\mathcal{M}=\langle\mathcal{F}, I\rangle$, where $\mathcal{F}=$ $\langle W, D, D o m, R\rangle$ is a frame and $I$ is a function taking names and predicates as arguments (an interpretation function). If $a$ is a name, then $I(a) \in D$. If $P$ is an $n$-place predicate, then $I(P): W \rightarrow \mathcal{P}\left(D^{n}\right)$. In particular, if $P$ is the identity predicate, then $I(P)(w)=\{\langle a, a\rangle: a \in D\}$ for all $w \in W$. A constant model of $\mathcal{L}_{\square}$ is a model $\mathcal{M}=\langle\mathcal{F}, I\rangle$ such that $\mathcal{F}$ is a constant frame.

The semantics for $\mathcal{L}_{\square}$ with respect to constant models is straightforward:

Definition 2.3 (Constant semantics) We say that, for any constant model $\mathcal{M}=\langle W, D$, Dom, $R, I\rangle$, world $w \in W$ and assignment function $g$ from the set of variables to $D$,

1. If $t$ is a name, then $\llbracket t \rrbracket_{\mathcal{M}, w, g}=I(t)$.

2. If $t$ is a variable, then $\llbracket t \rrbracket_{\mathcal{M}, w, g}=g(t)$.

3. If $t_{1}, \ldots, t_{n}$ are terms and $P$ is an $n$-place predicate, then we have $\llbracket P t_{1} \ldots t_{n} \rrbracket_{\mathcal{M}, w, g}$ $=1$ iff $\left\langle\llbracket t_{1} \rrbracket_{\mathcal{M}, w, g}, \ldots, \llbracket t_{n} \rrbracket_{\mathcal{M}, w, g}\right\rangle \in I(P)(w)$.

4. $\llbracket \neg \varphi \rrbracket_{\mathcal{M}, w, g}=1$ iff $\llbracket \varphi \rrbracket_{\mathcal{M}, w, g}=0$.

5. $\llbracket \varphi \wedge \psi \rrbracket_{\mathcal{M}, w, g}=1$ iff $\llbracket \varphi \rrbracket_{\mathcal{M}, w, g}=1$ and $\llbracket \psi \rrbracket_{\mathcal{M}, w, g}=1$.

6. $\llbracket \forall x \varphi \rrbracket \mathcal{M}, w, g=1$ iff, for all $d \in D, \llbracket \varphi \rrbracket_{\mathcal{M}, w, g[d / x]}=1$.

7. $\llbracket \square \varphi \rrbracket_{\mathcal{M}, w, g}=1$ iff, for all $w^{\prime} \in W$ s.t. $w R w^{\prime}, \llbracket \varphi \rrbracket_{\mathcal{M}, w^{\prime}, g}=1$.

where $g[d / x]$ is just like $g$ except that $g[d / x](x)=d$. For sentences, we say that $\llbracket \varphi \rrbracket_{\mathcal{M}, w}=1$ iff, for any assignment $g, \llbracket \varphi \rrbracket_{\mathcal{M}, w, g}=1$.

When it comes to non-constant models, we have to take account of cases where terms denote something outside the domain of the world of evaluation. How should we do that, assuming a classical approach? To answer this question, it's helpful to consider three-valued logic. Apart from the usual truth values 0 and 1, we introduce a third value $\mathrm{u}$, to be thought of as undefined. It will be assumed throughout that $\mathrm{u}$ does not belong to any domain of any model. We then introduce the following notions:

Definition 2.4 (Logical interpretations) A logical interpretation is a tuple $\mathrm{i}=$ $\left\langle i_{\neg}, i_{\wedge}, i_{\forall}, i_{\square}\right\rangle$, where 
1. $\mathrm{i}_{\neg}:\{0,1, \mathrm{u}\} \rightarrow\{0,1, \mathrm{u}\}$.

2. $\mathrm{i}_{\wedge}:\{0,1, \mathrm{u}\} \times\{0,1, \mathrm{u}\} \rightarrow\{0,1, \mathrm{u}\}$.

3. $\quad$ i $_{\forall}: \mathcal{P}(\{0,1, \mathrm{u}\}) \rightarrow\{0,1, \mathrm{u}\}$.

4. $\mathrm{i} \square: \mathcal{P}(\{0,1, \mathrm{u}\}) \rightarrow\{0,1, \mathrm{u}\}$.

Definition 2.5 (General semantics) Let $\mathrm{i}$ be a logical interpretation, and let $q, r \in$ $\{0,1, \mathrm{u}\}$. For any model $\mathcal{M}=\langle W, R, D$, Dom, $I\rangle$, world $w \in W$ and assignment $g$,

1. If $t$ is a name, then $\llbracket t \rrbracket_{\mathcal{M}, w, g}^{\mathrm{i}}=I(t)$.

2. If $t$ is a variable, then $\llbracket t \rrbracket_{\mathcal{M}, w, g}^{\mathrm{i}}=g(t)$.

3. If $x$ is a variable and $\varphi$ is a formula, then

(a) $\llbracket \iota x \varphi \rrbracket_{\mathcal{M}, w, g}^{\mathrm{i}}=d$ if

i. $\quad d \in \operatorname{Dom}(w)$ and $\llbracket \varphi \rrbracket_{\mathcal{M}, w, g[d / x]}^{\mathrm{i}}=1$, and

ii. for all $d^{\prime} \in \operatorname{Dom}(w)-\{d\}, \llbracket \varphi \rrbracket_{\mathcal{M}, w, g\left[d^{\prime} / x\right]}^{\mathrm{i}} \neq 1$.

(b) $\llbracket \iota x \varphi \rrbracket_{\mathcal{M}, w, g}^{\mathrm{i}}=\mathrm{u}$ otherwise.

4. If $t_{1}, \ldots, t_{n}$ are terms, $P$ is an $n$-place predicate and $X=\left\langle\llbracket t_{1} \rrbracket_{\mathcal{M}, w, g}^{\mathrm{i}}, \ldots\right.$, $\left.\llbracket t_{n} \rrbracket_{\mathcal{M}, w, g}^{\mathrm{i}}\right\rangle$, then

(a) If $X \in D^{n}$, then
i. $\llbracket P t_{1} \ldots t_{n} \rrbracket_{\mathcal{M}, w, g}^{\mathrm{i}}=1$ if $X \in \operatorname{Dom}(w)^{n}$ and $X \in I(P)(w)$.
ii. $\llbracket P t_{1} \ldots t_{n} \rrbracket_{\mathcal{M}, w, g}^{\mathrm{i}}=0$ if $X \in \operatorname{Dom}(w)^{n}$ and $X \notin I(P)(w)$.
iii. $\llbracket P t_{1} \ldots t_{n} \rrbracket_{\mathcal{M}, w, g}^{i}=p$ if $X \notin \operatorname{Dom}(w)^{n}$ and $X \in I(P)(w)$.
iv. $\llbracket P t_{1} \ldots t_{n} \rrbracket_{\mathcal{M}, w, g}^{\mathrm{i}}=q$ if $X \notin \operatorname{Dom}(w)^{n}$ and $X \notin I(P)(w)$.

(b) If $X \notin D^{n}$, then $\llbracket P t_{1} \ldots t_{n} \rrbracket_{\mathcal{M}, w, g}^{\mathrm{i}}=\mathrm{u}$.

5. $\llbracket \neg \varphi \rrbracket_{\mathcal{M}, w, g}^{\mathrm{i}}=\mathrm{i}_{\neg}\left(\llbracket \varphi \rrbracket_{\mathcal{M}, w, g}^{\mathrm{i}}\right)$.

6. $\llbracket \varphi \wedge \psi \rrbracket_{\mathcal{M}, w, g}^{\mathrm{i}}=\mathrm{i}_{\wedge}\left(\llbracket \varphi \rrbracket_{\mathcal{M}, w, g}^{\mathrm{i}}, \llbracket \psi \rrbracket_{\mathcal{M}, w, g}^{\mathrm{i}}\right)$

7. $\llbracket \forall x \varphi \rrbracket_{\mathcal{M}, w, g}^{\mathrm{i}}=\mathrm{i}_{\forall}\left(\left\{\llbracket \varphi \rrbracket_{\mathcal{M}, w, g[d / x]}^{\mathrm{i}}: d \in \operatorname{Dom}(w)\right\}\right)$

8. $\llbracket \square \varphi \rrbracket_{\mathcal{M}, w, g}^{\mathrm{i}}=\mathrm{i}_{\square}\left(\left\{\llbracket \varphi \rrbracket_{\mathcal{M}, w^{\prime}, g}^{\mathrm{i}}: w^{\prime} \in W\right.\right.$ s.t. $\left.\left.w R w^{\prime}\right\}\right)$

To see how this is supposed to work, consider the following example:

Example 2.1 Let $\mathcal{M}$ be a constant domain model with worlds $W=\left\{w_{1}, w_{2}\right\}$, domain $D=\left\{d_{1}, d_{2}\right\}$ and $R$ universal, and let $\mathrm{i}$ be a logical interpretation. Suppose $\varphi$ is a formula such that $\llbracket \varphi \rrbracket_{\mathcal{M}, w_{1}, g}^{\mathrm{i}}=1$ and $\llbracket \varphi \rrbracket_{\mathcal{M}, w_{2}, g}^{\mathrm{i}}=\mathrm{u}, \llbracket \varphi \rrbracket_{\mathcal{M}, w_{1}, g\left[d_{1} / x\right]}^{\mathrm{i}}=1$ and $\llbracket \varphi \rrbracket_{\mathcal{M}, w_{1}, g\left[d_{2} / x\right]}^{\mathrm{i}}=0$. Then $\llbracket \square \varphi \rrbracket_{\mathcal{M}, w_{1}, g}^{\mathrm{i}}=\mathrm{i}_{\square}(\{1, \mathrm{u}\})$ and $\llbracket \forall x \varphi \rrbracket_{\mathcal{M}, w_{1}, g}^{\mathrm{i}}=$ $\mathrm{i}_{\forall}(\{1,0\})$

For full generality, we should perhaps have assumed that $i_{\forall}$ and $i_{\square}$ are functions from the set of sequences of 0,1 and $u$ to $\{0,1, u\}$. Here, we make the simplifying assumption that the order and number of occurrences (above one) of a particular truth 
value doesn't matter. This assumptions is motivated by the fact that the corresponding assumption is made in the bivalent case.

The question we're most interested in is what the value of $p$ and $q$ should be. The route taken by for instance $[1, \mathrm{p}$. 102] is to let $p=1$ and $q=0$, yielding a total semantics. The route taken by [2, pp. 53-54] is to let $q=u .^{2}$ There are, however, at least seven other options to consider. In order to rule any of them out, we will need to have some idea of what a logical interpretation should be like. First of all, if the general semantics is going to agree with the constant semantics on the class of constant models, we have to require that the logical interpretations are normal, in the following sense:

Definition 2.6 (Normal logical interpretations) A logical interpretation i is normal iff the following obtains:

1. $\mathrm{i}_{\neg}(1)=0$ and $\mathrm{i}_{\neg}(0)=1$.

2. $\mathrm{i}_{\wedge}(1,1)=1$ and $\mathrm{i}_{\wedge}(1,0)=\mathrm{i}_{\wedge}(0,1)=\mathrm{i}_{\wedge}(0,0)=0$.

3. $i_{\forall}(\{1\})=1$ and $i_{\forall}(\{0\})=i_{\forall}(\{0,1\})=0$ and $i_{\forall}(\emptyset)=1$.

4. $\mathrm{i}_{\square}(\{1\})=1$ and $\mathrm{i}_{\square}(\{0\})=\mathrm{i}_{\square}(\{0,1\})=0$ and $\mathrm{i} \square(\emptyset)=1$.

To narrow down the list of logical interpretations further, it's reasonable to require that they respect certain classical equivalences. Unfortunately, however, it can be shown that no partial semantics respects every classical equivalence:

Fact 2.1 If $p=\mathrm{u}$ or $q=\mathrm{u}$, then there's no normal logical interpretation i such that, for every classically equivalent formula $\varphi$ and $\psi$ and every model $\mathcal{M}$ and world $w$, $\llbracket \varphi \rrbracket_{\mathcal{M}, w}^{\mathrm{i}}=\llbracket \psi \rrbracket_{\mathcal{M}, w}^{\mathrm{i}}$.

Proof Assume, toward contradiction, that $\mathrm{i}$ is normal and respects every classical equivalence. In particular, it satisfies principles $1-5$. As in the proof of Lemma A.1 in the Appendix, it follows that $\mathrm{i}_{\neg}(\mathrm{u})=\mathrm{u}, \mathrm{i}_{\wedge}(\mathrm{u}, \mathrm{u})=\mathrm{u}$ and $\mathrm{i}_{\wedge}(1, \mathrm{u})=\mathrm{u}$. Hence, with $\llbracket \varphi \rrbracket_{\mathcal{M}, w}^{\mathrm{i}}=1$ and $\llbracket \psi \rrbracket_{\mathcal{M}, w}^{\mathrm{i}}=\mathrm{u}$, we thus obtain $\llbracket \varphi \rrbracket_{\mathcal{M}, w}^{\mathrm{i}} \neq \llbracket \varphi \wedge \neg(\psi \wedge \neg \psi) \rrbracket_{\mathcal{M}, w}^{\mathrm{i}}=$ $\mathrm{u}$, contradicting our assumption.

In view of this fact, we shall prioritize equivalences with essentially the same nonlogical syntactic material present on both sides of the equation. Because intuitively, whatever syntactic input that gives rise to evaluation failure should also give rise to it in a classically equivalent configuration. We shall therefore require that logical interpretations are nice, in the following sense:

Definition 2.7 (Nice logical interpretations) A logical interpretation i is nice iff it's normal and, for every model $\mathcal{M}$, world $w$, assignment $g$ and formulas $\varphi, \psi, \theta$, the following 9 principles are satisfied:

1. $\llbracket \neg \neg \varphi \rrbracket_{\mathcal{M}, w, g}^{\mathrm{i}}=\llbracket \varphi \rrbracket_{\mathcal{M}, w, g}^{\mathrm{i}}$.

\footnotetext{
${ }^{2}$ Since they restrict the domain of interpretation to the domain of quantification for each world, the value of $p$ is irrelevant in their case.
} 
2. $\llbracket \varphi \wedge \psi \rrbracket_{\mathcal{M}, w, g}^{\mathrm{i}}=\llbracket \psi \wedge \varphi \rrbracket_{\mathcal{M}, w, g}^{\mathrm{i}}$.

3. $\llbracket \varphi \wedge \varphi \rrbracket_{\mathcal{M}, w, g}^{i}=\llbracket \varphi \rrbracket_{\mathcal{M}, w, g}^{i}$.

4. $\llbracket \varphi \wedge(\psi \wedge \theta) \rrbracket_{\mathcal{M}, w, g}^{i}=\llbracket(\varphi \wedge \psi) \wedge \theta \rrbracket_{\mathcal{M}, w, g}^{i}$.

5. $\llbracket(\varphi \wedge \theta) \vee(\psi \wedge \theta) \rrbracket_{\mathcal{M}, w, g}^{i}=\llbracket(\varphi \vee \psi) \wedge \theta \rrbracket_{\mathcal{M}, w, g}^{i}$.

6. If $\operatorname{Dom}(w) \neq \emptyset$ and $x$ isn't free in $\varphi$, then $\llbracket \forall x \varphi \rrbracket_{\mathcal{M}, w, g}^{\mathrm{i}}=\llbracket \varphi \rrbracket_{\mathcal{M}, w, g}^{\mathrm{i}}$.

7. $\llbracket \forall x(\varphi \wedge \psi) \rrbracket_{\mathcal{M}, w, g}^{\mathrm{i}}=\llbracket \forall x \varphi \wedge \forall x \psi \rrbracket_{\mathcal{M}, w, g}^{\mathrm{i}}$.

8. $\llbracket \square(\varphi \wedge \psi) \rrbracket_{\mathcal{M}, w, g}^{\mathrm{i}}=\llbracket \square \varphi \wedge \square \psi \rrbracket_{\mathcal{M}, w, g}^{\mathrm{i}}$.

9. If $W=\{w\}$ and $w R w$, then $\llbracket \square \varphi \rrbracket_{\mathcal{M}, w, g}^{i}=\llbracket \varphi \rrbracket_{\mathcal{M}, w, g}^{i}$.

We can now show the following:

Theorem 2.1 If $p=\mathrm{u}$ or $q=\mathrm{u}$, then the only nice logical interpretations are $\mathrm{w}$ and $\mathrm{s}$, defined as follows:

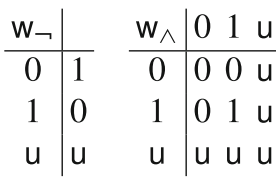

$$
\begin{aligned}
& \begin{array}{c|ccc|c}
\mathbf{w}_{\forall} & & \mathbf{w}_{\square} & \\
\hline\{0\} & 0 & & \{0\} & 0
\end{array} \\
& \{0,1\} \mid 0 \quad\{0,1\} 0 \\
& \{1\} \quad 1 \quad\{1\} \mid 1 \\
& \{u\} u \quad\{u\} u \\
& \{0, u\} u \quad\{0, u\} u \\
& \{1, u\} u \quad\{1, u\} u \\
& \{0,1, u\} \cup \quad\{0,1, u\} u \\
& \emptyset|1 \quad \emptyset| 1
\end{aligned}
$$

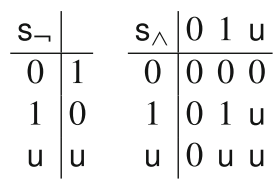

$$
\begin{aligned}
& \begin{array}{r|c}
s_{\forall} & \\
\hline\{0\} & 0
\end{array} \\
& \{0,1\} 0 \\
& \{1\} 1 \\
& \{u\} \mathrm{u} \\
& \{0, u\} 0 \\
& \{1, u\} \mathrm{u} \\
& \{0,1, u\} 0 \\
& \varnothing 1 \\
& \begin{array}{r|c}
\mathrm{s} \square & \\
\hline\{0\} & 0 \\
\{0,1\} & 0 \\
\{1\} & 1 \\
\{u\} & \mathrm{u} \\
\{0, \mathrm{u}\} & 0 \\
\{1, u\} & \mathrm{u} \\
\{0,1, u\} & 0 \\
\varnothing & 1
\end{array}
\end{aligned}
$$

For a proof, see Appendix A.1. As indicated by the choice of letters, $w$ is an extension of Kleene's weak three-valued propositional logic, whereas $s$ is an extension of the strong one.

Finally, we are in a position so say something about the value of $q$. For [3], the point of introducing varying domain semantics was to invalidate the so called Barcan formula:

(3) $\forall x \square \varphi \rightarrow \square \forall x \varphi$.

The Barcan formula, and its converse, are both valid on the constant domain semantics. But the validity of the Barcan formula and its converse is only problematic if one reads $\forall$ as for every existing object rather than for every possible object. In other words, if one believes that it's possible to make claims about existence without 
using an existence predicate. Arguably, the point of using varying domains is to be able to do just that. In particular, to be able to truthfully say things like

(4) Everything is such that it could have failed to exist, although it's necessary that something exists.

if such be the case, using only quantifiers and the identity predicate, respectively:

(5) $\forall x \diamond \neg \exists y(y=x) \wedge \square \exists x(x=x)$.

But in order for this to be possible, we have to require that $q=0$. Because if $q \neq 0$ and $\mathrm{i} \in\{\mathrm{w}, \mathrm{s}\},(5)$ will never be true (see Fact A.1 in the Appendix).

As for the value of $p$, this is only relevant if one has a model where the extension of a predicate in a world contain things not existing in that world. Presumably, the point of allowing such models is to to be able to truthfully say things like

(6) Someone exists that could have been dead, although it's necessary that everyone who exists isn't dead.

i.e.

(7) $\exists x \diamond P x \wedge \square \forall x \neg P x$.

In order for this to be possible when $q=0$ and $\mathrm{i} \in\{\mathrm{w}, \mathrm{s}\}$, we have to require that $p=1$ (see Fact A.2 in the Appendix). All in all, this leaves us with only two possible partial semantics for $\mathcal{L}_{\square}$, namely the ones given in Definition 2.5 when $p=1$, $q=0$ and $\mathrm{i} \in\{\mathrm{w}, \mathrm{s}\}$.

\section{Conclusion}

When it comes to partial semantics, there's no way of preserving every classical equivalence between formulas (Fact 2.1). If one has to choose, it seems reasonable to prioritize equivalences where essentially the same syntactic material is present on both sides of the equation. Doing so yields two possible interpretations of the logical operators, which are extensions of Kleene's weak and strong semantics for propositional logic, respectively (Theorem 2.1). This is our main result. Provided that the point of using varying domains is to be able to make certain true claims about existence, e.g. (4) and (6), without using any existence predicate, this yields a total of two possible partial semantics for quantified modal logic, a weak and a strong one.

Acknowledgments I want to thank Graeme Forbes, Peter Pagin, Dag Westerståhl and the participants of the November 252016 CLLAM seminar at the Department of Philosophy at Stockholm University for comments on earlier versions of this paper. Especially, I want to thank an anonymous referee for comments and suggestions during the review process.

Open Access This article is distributed under the terms of the Creative Commons Attribution 4.0 International License (http://creativecommons.org/licenses/by/4.0/), which permits unrestricted use, distribution, and reproduction in any medium, provided you give appropriate credit to the original author(s) and the source, provide a link to the Creative Commons license, and indicate if changes were made. 


\section{Appendix}

\section{A.1 Nice Logical Interpretations}

We prove Theorem 2.1.

Lemma A.1 If $\mathrm{i}$ is nice and $p=\mathrm{u}$ or $q=\mathrm{u}$, then $\mathrm{i}_{\neg}=\mathrm{w}_{\neg}=\mathrm{s}_{\neg}$ and either $\mathrm{i}_{\wedge}=\mathrm{w}_{\wedge}$ or $\mathrm{i}_{\wedge}=\mathrm{s}_{\wedge}$.

Proof Assume that $\mathrm{i}$ is nice. If $q=\mathrm{u}$, let $\mathcal{M}=\langle W, D, D o m, R, I\rangle$ be a model such that $W=\{w\}, D=\{d, e\}, \operatorname{Dom}(w)=\{d\}$ and $I(P)(w)=\{d\}$. If $p=\mathrm{u}$, let $I(P)(w)=D$ instead. Let $g$ be an assignment such that $g(x)=d$ and $g(y)=e$. In either case, we have $\llbracket P x \rrbracket_{\mathcal{M}, w, g}^{\mathrm{i}}=1, \llbracket \neg P x \rrbracket_{\mathcal{M}, w, g}^{\mathrm{i}}=0$ and $\llbracket P y \rrbracket_{\mathcal{M}, w, g}^{\mathrm{i}}=\mathrm{u}$.

Let $\varphi$ be $P x$ and $\psi$ be $P y$. By principle $1, \mathrm{i}_{\neg}=\mathrm{w}_{\neg}=\mathrm{s}_{\neg}$. Otherwise we get $\llbracket \neg \neg \psi \rrbracket_{\mathcal{M}, w, g}^{\mathrm{i}} \neq \llbracket \psi \rrbracket_{\mathcal{M}, w, g}^{\mathrm{i}}$. By principle $3, \mathrm{i}_{\wedge}(\mathrm{u}, \mathrm{u})=\mathrm{u}$. Otherwise we get $\llbracket \psi \wedge \psi \rrbracket_{\mathcal{M}, w, g}^{\mathrm{i}} \neq \llbracket \psi \rrbracket_{\mathcal{M}, w, g}^{\mathrm{i}}$. By principle $2, \mathrm{i}_{\wedge}(a, b)=\mathrm{i}_{\wedge}(b, a)$ for any $a, b \in$ $\{0,1, \mathrm{u}\}$. Otherwise we either get $\llbracket \varphi \wedge \psi \rrbracket_{\mathcal{M}, w, g}^{\mathrm{i}} \neq \llbracket \psi \wedge \varphi \rrbracket_{\mathcal{M}, w, g}^{\mathrm{i}}$ or $\llbracket \neg \varphi \wedge$ $\psi \rrbracket_{\mathcal{M}, w, g}^{\mathrm{i}} \neq \llbracket \psi \wedge \neg \varphi \rrbracket_{\mathcal{M}, w, g}^{\mathrm{i}}$. By principle $4, \mathrm{i}_{\wedge}(0, \mathrm{u}) \neq 1$. Otherwise $\llbracket \neg \varphi \wedge(\neg \varphi \wedge$ $\psi) \rrbracket_{\mathcal{M}, w, g}^{\mathrm{i}} \neq \llbracket(\neg \varphi \wedge \neg \varphi) \wedge \psi \rrbracket_{\mathcal{M}, w, g}^{\mathrm{i}}$. Also, by principle 4 , if $\mathrm{i}_{\wedge}(0, \mathrm{u})=\mathrm{u}$, it's required that $\mathrm{i}_{\wedge}(1, \mathrm{u})=\mathrm{u}$. Otherwise we get $\llbracket \neg \varphi \wedge(\varphi \wedge \psi) \rrbracket_{\mathcal{M}, w, g}^{\mathrm{i}} \neq \llbracket(\neg \varphi \wedge$ $\varphi) \wedge \psi \rrbracket_{\mathcal{M}, w, g}^{\mathrm{i}}$. Finally, by principle 5 , if $\mathrm{i}_{\wedge}(0, \mathrm{u})=0$, then $\mathrm{i}_{\wedge}(1, \mathrm{u})=\mathrm{u}$. Otherwise $\llbracket(\neg \varphi \wedge \varphi) \vee(\psi \wedge \varphi) \rrbracket_{\mathcal{M}, w, g}^{\mathrm{i}} \neq \llbracket(\neg \varphi \vee \psi) \wedge \varphi \rrbracket_{\mathcal{M}, w, g}^{\mathrm{i}}$. Hence, $\mathrm{i}_{\wedge}=\mathrm{w}_{\wedge}$ or $\mathrm{i}_{\wedge}=\mathbf{s}_{\wedge}$.

Lemma A.2 If $\mathrm{i}$ is nice, then

1. if $\mathrm{i}_{\wedge}=\mathrm{w}_{\wedge}$, then $\mathrm{i}_{\forall}=\mathrm{w}_{\forall}$.

2. if $\mathrm{i}_{\wedge}=\mathrm{s}_{\wedge}$, then $\mathrm{i}_{\forall}=\mathrm{s}_{\forall}$ and $\mathrm{i}_{\square}(\{\mathrm{u}\})=\mathrm{u}$.

Proof Assume that $\mathrm{i}$ is nice. Let $\mathcal{M}=\langle W, D$, Dom, $R, I\rangle$ be a model such that

- $W=\{w\}, D=\left\{d_{1}, d_{2}, d_{3}\right\}, \operatorname{Dom}(w)=D, R=W^{2}$.

- $I(A)(w)=\emptyset$.

- $\quad I(F)(w)=\left\{d_{3}\right\}, I(G)(w)=\left\{d_{2}, d_{3}\right\}$ and $I(H)(w)=D$.

- $\quad I(P)(w)=\left\{\left\langle d_{1}, d_{1}\right\rangle,\left\langle d_{2}, d_{2}\right\rangle\right\}$ and $I(Q)(w)=\left\{\left\langle d_{3}, d_{3}\right\rangle\right\}$.

Then, for any assignment $g$,

- $\llbracket F x \rrbracket_{\mathcal{M}, w, g\left[d_{1} / x\right]}^{\mathrm{i}}=0, \llbracket F x \rrbracket_{\mathcal{M}, w, g\left[d_{2} / x\right]}^{\mathrm{i}}=0, \llbracket F x \rrbracket_{\mathcal{M}, w, g\left[d_{3} / x\right]}^{\mathrm{i}}=1$.

- $\llbracket G x \rrbracket_{\mathcal{M}, w, g\left[d_{1} / x\right]}^{\mathrm{i}}=0, \llbracket G x \rrbracket_{\mathcal{M}, w, g\left[d_{2} / x\right]}^{\mathrm{i}}=1, \llbracket G x \rrbracket_{\mathcal{M}, w, g\left[d_{3} / x\right]}^{\mathrm{i}}=1$.

- $\llbracket H(\iota y P x y) \rrbracket_{\mathcal{M}, w, g\left[d_{1} / x\right]}^{\mathrm{i}}=1, \llbracket H(\iota y P x y) \rrbracket_{\mathcal{M}, w, g\left[d_{2} / x\right]}^{\mathrm{i}}=1$, $\llbracket H(\iota y P x y) \rrbracket_{\mathcal{M}, w, g\left[d_{3} / x\right]}^{\mathrm{i}}=\mathrm{u}$.

- $\llbracket H(\iota y Q x y) \rrbracket_{\mathcal{M}, w, g\left[d_{1} / x\right]}^{\mathrm{i}}=\mathrm{u}, \llbracket H(\iota y Q x y) \rrbracket_{\mathcal{M}, w, g\left[d_{2} / x\right]}^{\mathrm{i}}=\mathrm{u}$, $\llbracket H(\iota y Q x y) \rrbracket_{\mathcal{M}, w, g\left[d_{3} / x\right]}^{\mathrm{i}}=1$.

- $\llbracket H(\iota x A x) \rrbracket_{\mathcal{M}, w, g\left[d_{1} / x\right]}^{\mathrm{i}}=\mathrm{u}, \llbracket H(\iota x A x) \rrbracket_{\mathcal{M}, w, g\left[d_{2} / x\right]}^{\mathrm{i}}=\mathrm{u}$, 


$$
\llbracket H(\iota x A x) \rrbracket_{\mathcal{M}, w, g\left[d_{3} / x\right]}^{\mathrm{i}}=\mathrm{u} .
$$

Let

- $\varphi_{1}=F x$ and $\psi_{1}=H(\iota y P x y)$.

- $\varphi_{2}=G x$ and $\psi_{2}=H(\iota y P x y)$.

- $\varphi_{3}=H(\iota y P x y)$ and $\psi_{3}=H(\iota y Q x y)$.

- $\theta=H(\iota x A x)$.

By principle 6, $\mathrm{i}_{\forall}(\{\mathrm{u}\})=\mathrm{u}$. Otherwise $\llbracket \forall x \theta \rrbracket_{\mathcal{M}, w, g}^{\mathrm{i}} \neq \llbracket \theta \rrbracket_{\mathcal{M}, w, g}^{\mathrm{i}}$. By principle 7, $i_{\forall}(\{\mathbf{u}\})=i_{\forall}(\{1, \mathbf{u}\})$. Otherwise $\llbracket \forall x\left(\varphi_{3} \wedge \psi_{3}\right) \rrbracket_{\mathcal{M}, w, g}^{i} \neq \llbracket \forall x \varphi_{3} \wedge \forall x \psi_{3} \rrbracket_{\mathcal{M}, w, g}^{i}$. By Lemma A.1, there are two cases to consider:

1. $\mathrm{i}_{\wedge}=\mathrm{w}_{\wedge}$. By principle $7, \mathrm{i}_{\forall}(\{0, \mathrm{u}\})=\mathrm{u}$ and $\mathrm{i}_{\forall}(\{0,1, \mathrm{u}\})=\mathrm{u}$. Otherwise $\llbracket \forall x\left(\varphi_{1} \wedge \psi_{1}\right) \rrbracket_{\mathcal{M}, w, g}^{i} \neq \llbracket \forall x \varphi_{1} \wedge \forall x \psi_{1} \rrbracket_{\mathcal{M}, w, g}^{i}$ or $\llbracket \forall x\left(\varphi_{2} \wedge \psi_{2}\right) \rrbracket_{\mathcal{M}, w, g}^{i} \neq$ $\llbracket \forall x \varphi_{2} \wedge \forall x \psi_{2} \rrbracket_{\mathcal{M}, w, g}^{i}$. Hence, $i_{\forall}=w_{\forall}$.

2. $\mathrm{i}_{\wedge}=\mathrm{s}_{\wedge}$. By principle $7, \mathrm{i}_{\forall}(\{0, \mathrm{u}\})=0$ and $\mathrm{i}_{\forall}(\{0,1, \mathrm{u}\})=0$. Otherwise $\llbracket \forall x\left(\varphi_{1} \wedge \psi_{1}\right) \rrbracket_{\mathcal{M}, w, g}^{i} \neq \llbracket \forall x \varphi_{1} \wedge \forall x \psi_{1} \rrbracket_{\mathcal{M}, w, g}^{i}$ or $\llbracket \forall x\left(\varphi_{2} \wedge \psi_{2}\right) \rrbracket_{\mathcal{M}, w, g}^{i} \neq$ $\llbracket \forall x \varphi_{2} \wedge \forall x \psi_{2} \rrbracket_{\mathcal{M}, w, g}^{i}$. Hence, $i_{\forall}=s_{\forall}$.

Finally, by principle $9, \mathrm{i} \square(\{u\})=$ u. Otherwise $\llbracket \square \theta \rrbracket_{\mathcal{M}, w, g}^{i} \neq \llbracket \theta \rrbracket_{\mathcal{M}, w, g}^{i}$.

Lemma A.3 If $\mathrm{i}$ is nice and $p=\mathrm{u}$ or $q=\mathrm{u}$, then

1. if $\mathrm{i}_{\wedge}=\mathrm{w}_{\wedge}$, then $\mathrm{i}_{\square}=\mathrm{w}_{\square}$.

2. if $\mathrm{i}_{\wedge}=\mathrm{s}_{\wedge}$, then $\mathrm{i}_{\square}=\mathrm{s}_{\square}$.

Proof Assume that $\mathrm{i}$ is nice and that $\mathrm{i}_{\wedge}=\mathrm{w}_{\wedge}$ or $\mathrm{i}_{\wedge}=\mathrm{s}_{\wedge}$. By Lemma A.1, $\mathrm{i}_{\neg}=\mathrm{w}_{\neg}=\mathrm{s}_{\neg}$. By Lemma A.2, $\mathrm{i}_{\square}(\{1, \mathrm{u}\})=\mathrm{u}$. Now, if $q=\mathrm{u}$, consider a model $\mathcal{M}=\langle W, D$, Dom, $R, I\rangle$ such that

- $W=\left\{w_{1}, w_{2}, w_{3}\right\}, D=\left\{d_{1}, d_{2}, d_{3}\right\}, \operatorname{Dom}\left(w_{1}\right)=\left\{d_{1}, d_{2}\right\}, \operatorname{Dom}\left(w_{2}\right)=$ $\left\{d_{1}, d_{2}\right\}, \operatorname{Dom}\left(w_{3}\right)=\left\{d_{1}, d_{3}\right\}, R=W^{2}$.

- $I\left(c_{1}\right)=d_{1}, I\left(c_{2}\right)=d_{2}, I\left(c_{3}\right)=d_{3}$.

- $I(F)\left(w_{1}\right)=\emptyset, I(F)\left(w_{2}\right)=\emptyset, I(F)\left(w_{3}\right)=\operatorname{Dom}\left(w_{3}\right)$.

- $I(G)\left(w_{1}\right)=\emptyset, I(G)\left(w_{2}\right)=\operatorname{Dom}\left(w_{2}\right), I(G)\left(w_{3}\right)=\operatorname{Dom}\left(w_{3}\right)$.

- $I(H)\left(w_{1}\right)=\operatorname{Dom}\left(w_{1}\right), I(H)\left(w_{2}\right)=\operatorname{Dom}\left(w_{2}\right), I(H)\left(w_{3}\right)=\operatorname{Dom}\left(w_{3}\right)$.

If $p=\mathrm{u}$, let $I(H)\left(w_{1}\right)=I(H)\left(w_{2}\right)=I(H)\left(w_{3}\right)=D$ instead. In either case, for any assignment $g$, it follows by Lemma A.1 that

- $\llbracket F c_{1} \rrbracket_{\mathcal{M}, w_{1}, g}^{i}=0, \llbracket F c_{1} \rrbracket_{\mathcal{M}, w_{2}, g}^{i}=0, \llbracket F c_{1} \rrbracket_{\mathcal{M}, w_{3}, g}^{i}=1$.

- $\llbracket G c_{1} \rrbracket_{\mathcal{M}, w_{1}, g}^{\mathrm{i}}=0, \llbracket G c_{1} \rrbracket_{\mathcal{M}, w_{2}, g}^{\mathrm{i}}=1, \llbracket G c_{1} \rrbracket_{\mathcal{M}, w_{3}, g}^{\mathrm{i}}=1$.

- $\llbracket H c_{2} \rrbracket_{\mathcal{M}, w_{1}, g}^{\mathrm{i}}=1, \llbracket H c_{2} \rrbracket_{\mathcal{M}, w_{2}, g}^{\mathrm{i}}=1, \llbracket H c_{2} \rrbracket_{\mathcal{M}, w_{3}, g}^{\mathrm{i}}=\mathrm{u}$.

- $\llbracket H c_{3} \rrbracket_{\mathcal{M}, w_{1}, g}^{\mathrm{i}}=\mathrm{u}, \llbracket H c_{3} \rrbracket_{\mathcal{M}, w_{2}, g}^{\mathrm{i}}=\mathrm{u}, \llbracket H c_{3} \rrbracket_{\mathcal{M}, w_{3}, g}^{\mathrm{i}}=1$.

- $\llbracket \neg H c_{2} \rrbracket_{\mathcal{M}, w_{1}, g}^{i_{1}}=0, \llbracket \neg H c_{2} \rrbracket_{\mathcal{M}, w_{2}, g}^{i_{2}}=0, \llbracket \neg H c_{2} \rrbracket_{\mathcal{M}, w_{3}, g}^{i}=u$.

- $\llbracket H c_{2} \wedge H c_{3} \rrbracket_{\mathcal{M}, w_{1}, g}^{i}=\mathrm{u}, \llbracket H c_{2} \wedge H c_{3} \rrbracket_{\mathcal{M}, w_{2}, g}^{\mathrm{i}}=\mathrm{u}, \llbracket H c_{2} \wedge H c_{3} \rrbracket_{\mathcal{M}, w_{3}, g}^{\mathrm{i}}=\mathrm{u}$. 
Let

- $\varphi_{1}=F c_{1}$ and $\psi_{1}=H c_{2}$.

- $\varphi_{2}=G c_{1}$ and $\psi_{2}=H c_{2}$.

- $\varphi_{3}=H c_{2}$ and $\psi_{3}=H c_{3}$.

- $\varphi_{4}=\neg H c_{2}$ and $\psi_{4}=H c_{2} \wedge H c_{3}$.

First we observe that, by principle $8, \mathrm{i}_{\square}(\{\mathrm{u}\})=\mathrm{i}_{\square}(\{1, \mathrm{u}\})$. Otherwise we get $\llbracket \square\left(\varphi_{3} \wedge\right.$ $\left.\psi_{3}\right) \rrbracket_{\mathcal{M}, w_{1}, g}^{\mathrm{i}} \neq \llbracket \square \varphi_{3} \wedge \square \psi_{3} \rrbracket_{\mathcal{M}, w_{1}, g}^{\mathrm{i}}$. Next, we consider each case in turn:

1. Assume that $\mathrm{i}_{\wedge}=\mathrm{w}_{\wedge}$. By principle $8, \mathrm{i}_{\square}(\{0, \mathrm{u}\})=\mathrm{u}$ and $\mathrm{i}_{\square}(\{0,1, \mathrm{u}\})=\mathrm{u}$. Otherwise $\llbracket \square\left(\varphi_{1} \wedge \psi_{1}\right) \rrbracket_{\mathcal{M}, w_{1}, g}^{\mathrm{i}} \neq \llbracket \square \varphi_{1} \wedge \square \psi_{1} \rrbracket_{\mathcal{M}, w_{1}, g}^{\mathrm{i}}$ or $\llbracket \square\left(\varphi_{2} \wedge \psi_{2}\right) \rrbracket_{\mathcal{M}, w_{1}, g}^{\mathrm{i}} \neq$ $\llbracket \square \varphi_{2} \wedge \square \psi_{2} \rrbracket_{\mathcal{M}, w_{1}, g}^{\mathrm{i}}$. From $\mathrm{i}_{\square}(\{0, \mathrm{u}\})=\mathrm{u}$, it follows by principle 8 that $\mathrm{i} \square(\{\mathrm{u}\})=\mathrm{u}$. Otherwise $\llbracket \square\left(\varphi_{4} \wedge \psi_{4}\right) \rrbracket_{\mathcal{M}, w_{1}, g}^{\mathrm{i}} \neq \llbracket \square \varphi_{4} \wedge \square \psi_{4} \rrbracket_{\mathcal{M}, w_{1}, g}^{\mathrm{i}}$. Hence, $\mathrm{i} \square=\mathrm{w}_{\square}$.

2. Assume that $\mathrm{i}_{\wedge}=\mathrm{s}_{\wedge}$. By Lemma A.2, $\mathrm{i}_{\square}(\{\mathrm{u}\})=\mathrm{u}$. By principle 8 , $\mathrm{i}_{\square}(\{0, \mathrm{u}\})=$ 0 and $\mathrm{i}_{\square}(\{0,1, \mathrm{u}\})=0$. Otherwise $\llbracket \square\left(\varphi_{1} \wedge \psi_{1}\right) \rrbracket_{\mathcal{M}, w_{1}, g}^{\mathrm{i}} \neq \llbracket \square \varphi_{1} \wedge \square \psi_{1} \rrbracket_{\mathcal{M}, w_{1}, g}^{\mathrm{i}}$ or $\llbracket \square\left(\varphi_{2} \wedge \psi_{2}\right) \rrbracket_{\mathcal{M}, w_{1}, g}^{\mathrm{i}} \neq \llbracket \square \varphi_{2} \wedge \square \psi_{2} \rrbracket_{\mathcal{M}, w_{1}, g}^{\mathrm{i}}$. Hence, $\mathrm{i} \square=\mathrm{s}_{\square}$.

Lemma A.4 $\mathrm{w}$ and $\mathrm{s}$ are nice.

Proof $\mathrm{w}$ and s obviously satisfy principles $1-3$ and 9 . As for principles 4 and 5, let $\mathrm{w}_{\vee}(a, b)={ }_{d f} \mathrm{w}_{\neg}\left(\mathrm{w}_{\wedge}\left(\mathrm{w}_{\neg}(a), \mathrm{w}_{\neg}(b)\right)\right)$ and $\mathrm{s}_{\vee}(a, b)={ }_{d f} \mathrm{~s}_{\neg}\left(\mathrm{s}_{\wedge}\left(\mathrm{s}_{\neg}(a), \mathrm{s}_{\neg}(b)\right)\right)$. It's then easy to verify that, for each $a, b, c \in\{0,1, \mathrm{u}\}$,

1. (a) $\mathrm{w}_{\wedge}\left(a, \mathrm{w}_{\wedge}(b, c)\right)=\mathrm{w}_{\wedge}\left(\mathrm{w}_{\wedge}(a, b), c\right)$.

(b) $\mathrm{s}_{\wedge}\left(a, \mathrm{~s}_{\wedge}(b, c)\right)=\mathrm{s}_{\wedge}\left(\mathrm{s}_{\wedge}(a, b), c\right)$.

2. (a) $\mathrm{w}_{\vee}\left(\mathrm{w}_{\wedge}(a, c), \mathrm{w}_{\wedge}(b, c)\right)=\mathrm{w}_{\wedge}\left(\mathrm{w}_{\vee}(a, b), c\right)$.

(b) $\mathrm{s}_{\vee}\left(\mathrm{s}_{\wedge}(a, c), \mathrm{s}_{\wedge}(b, c)\right)=\mathrm{s}_{\wedge}\left(\mathrm{s}_{\vee}(a, b), c\right)$.

As for principle 7, we consider each interpretation in turn:

1. Suppose (i) $\llbracket \forall x(\varphi \wedge \psi) \rrbracket_{\mathcal{M}, w, g}^{\mathrm{w}}=0$ but (ii) $\llbracket \forall x \varphi \wedge \forall x \psi \rrbracket_{\mathcal{M}, w, g}^{\mathrm{w}}=1$. By (ii), $\llbracket \forall x \varphi \rrbracket_{\mathcal{M}, w, g}^{\mathrm{w}}=1$ and $\llbracket \forall x \psi \rrbracket_{\mathcal{M}, w, g}^{\mathrm{w}}=1$. Hence, we have $\llbracket \varphi \rrbracket_{\mathcal{M}, w, g[d / x]}^{\mathrm{w}}=1$ and $\llbracket \psi \rrbracket_{\mathcal{M}, w, g[d / x]}^{\mathrm{w}}=1$ for all $d \in \operatorname{Dom}(w)$. Hence, $\llbracket \varphi \wedge \psi \rrbracket_{\mathcal{M}, w, g[d / x]}^{\mathrm{w}}=1$ for all $d \in \operatorname{Dom}(w)$, contradicting (i).

Suppose (i) $\llbracket \forall x(\varphi \wedge \psi) \rrbracket_{\mathcal{M}, w, g}^{\mathrm{w}}=0$ but (ii) $\llbracket \forall x \varphi \wedge \forall x \psi \rrbracket_{\mathcal{M}, w, g}^{\mathrm{w}}=\mathrm{u}$. By (ii), $\llbracket \forall x \varphi \rrbracket_{\mathcal{M}, w, g}^{\mathrm{w}}=\mathrm{u}$ or $\llbracket \forall x \psi \rrbracket_{\mathcal{M}, w, g}^{\mathrm{w}}=\mathrm{u}$. Hence, $\llbracket \varphi \rrbracket_{\mathcal{M}, w, g[d / x]}^{\mathrm{w}}=\mathrm{u}$ for some $d \in$ $\operatorname{Dom}(w)$ or $\llbracket \psi \rrbracket_{\mathcal{M}, w, g[d / x]}^{\mathrm{w}}=\mathrm{u}$ for some $d \in \operatorname{Dom}(w)$. Hence, $\llbracket \varphi \wedge \psi \rrbracket_{\mathcal{M}, w, g}^{\mathrm{w}}=$ $\mathrm{u}$ for some $d \in \operatorname{Dom}(w)$, contradicting (i).

Suppose (i) $\llbracket \forall x(\varphi \wedge \psi) \rrbracket_{\mathcal{M}, w, g}^{\mathrm{w}}=1$ but (ii) $\llbracket \forall x \varphi \wedge \forall x \psi \rrbracket_{\mathcal{M}, w, g}^{\mathrm{w}}=0$. By (ii), $\llbracket \forall x \varphi \rrbracket_{\mathcal{M}, w, g}^{\mathrm{W}}=0$ or $\llbracket \forall x \psi \rrbracket_{\mathcal{M}, w, g}^{\mathrm{W}}=0$. Hence, $\llbracket \varphi \rrbracket_{\mathcal{M}, w, g[d / x]}^{\mathrm{W}}=0$ for some $d \in \operatorname{Dom}(w)$ or $\llbracket \psi \rrbracket_{\mathcal{M}, w, g[d / x]}^{w}=0$ for some $d \in \operatorname{Dom}(w)$. Hence, $\llbracket \varphi \wedge$ $\psi \rrbracket_{\mathcal{M}, w, g[d / x]}^{\mathrm{w}} \neq 1$ for some $d \in \operatorname{Dom}(w)$, contradicting (i).

Suppose (i) $\llbracket \forall x(\varphi \wedge \psi) \rrbracket_{\mathcal{M}, w, g}^{\mathrm{w}}=1$ but (ii) $\llbracket \forall x \varphi \wedge \forall x \psi \rrbracket_{\mathcal{M}, w, g}^{\mathrm{w}}=\mathrm{u}$. By (ii), $\llbracket \forall x \varphi \rrbracket_{\mathcal{M}, w, g}^{\mathrm{w}}=\mathrm{u}$ or $\llbracket \forall x \psi \rrbracket_{\mathcal{M}, w, g}^{\mathrm{w}}=\mathrm{u}$. Hence, $\llbracket \varphi \rrbracket_{\mathcal{M}, w, g[d / x]}^{\mathrm{w}}=\mathbf{u}$ for 
some $d \in \operatorname{Dom}(w)$ or $\llbracket \psi \rrbracket_{\mathcal{M}, w, g[d / x]}^{\mathrm{w}}=\mathrm{u}$ for some $d \in \operatorname{Dom}(w)$. Hence, $\llbracket \varphi \wedge$ $\psi \rrbracket_{\mathcal{M}, w, g[d / x]}^{\mathrm{w}}=\mathrm{u}$ for some $d \in \operatorname{Dom}(w)$, contradicting (i).

Suppose (i) $\llbracket \forall x(\varphi \wedge \psi) \rrbracket_{\mathcal{M}, w, g}^{\mathrm{w}}=\mathrm{u}$ but (ii) $\llbracket \forall x \varphi \wedge \forall x \psi \rrbracket_{\mathcal{M}, w, g}^{\mathrm{w}}=0$. By (ii), $\llbracket \forall x \varphi \rrbracket_{\mathcal{M}, w, g}^{\mathrm{w}} \neq \mathrm{u}$ and $\llbracket \forall x \psi \rrbracket_{\mathcal{M}, w, g}^{\mathrm{w}} \neq \mathrm{u}$. Hence, we have $\llbracket \varphi \rrbracket_{\mathcal{M}, w, g[d / x]}^{\mathrm{w}} \neq \mathrm{u}$ and $\llbracket \psi \rrbracket_{\mathcal{M}, w, g[d / x]}^{\mathrm{w}} \neq \mathrm{u}$ for all $d \in \operatorname{Dom}(w)$. Hence, $\llbracket \varphi \wedge \psi \rrbracket_{\mathcal{M}, w, g[d / x]}^{\mathrm{w}} \neq \mathrm{u}$ for all $d \in \operatorname{Dom}(w)$, contradicting (i).

Suppose (i) $\llbracket \forall x(\varphi \wedge \psi) \rrbracket_{\mathcal{M}, w, g}^{\mathrm{w}}=\mathrm{u}$ but (ii) $\llbracket \forall x \varphi \wedge \forall x \psi \rrbracket_{\mathcal{M}, w, g}^{\mathrm{w}}=1$. By (ii), $\llbracket \forall x \varphi \rrbracket_{\mathcal{M}, w, g}^{\mathrm{w}}=1$ and $\llbracket \forall x \psi \rrbracket_{\mathcal{M}, w, g}^{\mathrm{w}}=1$. Hence, we have $\llbracket \varphi \rrbracket_{\mathcal{M}, w, g[d / x]}^{\mathrm{w}}=1$ and $\llbracket \psi \rrbracket_{\mathcal{M}, w, g[d / x]}^{\mathrm{W}}=1$ for all $d \in \operatorname{Dom}(w)$. Hence, $\llbracket \varphi \wedge \psi \rrbracket_{\mathcal{M}, w, g[d / x]}^{\mathrm{W}}=1$ for all $d \in \operatorname{Dom}(w)$, contradicting (i).

2. Suppose (i) $\llbracket \forall x(\varphi \wedge \psi) \rrbracket_{\mathcal{M}, w, g}^{\mathrm{s}}=0$ but (ii) $\llbracket \forall x \varphi \wedge \forall x \psi \rrbracket_{\mathcal{M}, w, g}^{\mathrm{s}}=1$. By (ii), $\llbracket \forall x \varphi \rrbracket_{\mathcal{M}, w, g}^{\mathrm{s}}=1$ and $\llbracket \forall x \psi \rrbracket_{\mathcal{M}, w, g}^{\mathrm{s}}=1$. Hence, we have $\llbracket \varphi \rrbracket_{\mathcal{M}, w, g[d / x]}^{\mathrm{s}^{\mathrm{s}}}=1$ and $\llbracket \psi \rrbracket_{\mathcal{M}, w, g[d / x]}^{\mathrm{s}}=1$ for all $d \in \operatorname{Dom}(w)$. Hence, $\llbracket \varphi \wedge \psi \rrbracket_{\mathcal{M}, w, g[d / x]}^{\mathrm{s}}=1$ for all $d \in \operatorname{Dom}(w)$, contradicting (i).

Suppose (i) $\llbracket \forall x(\varphi \wedge \psi) \rrbracket_{\mathcal{M}, w, g}^{\mathrm{s}}=0$ but (ii) $\llbracket \forall x \varphi \wedge \forall x \psi \rrbracket_{\mathcal{M}, w, g}^{\mathrm{s}}=\mathrm{u}$. By (ii), $\llbracket \forall x \varphi \rrbracket_{\mathcal{M}, w, g}^{\mathrm{s}} \neq 0$ and $\llbracket \forall x \psi \rrbracket_{\mathcal{M}, w, g}^{\mathrm{s}} \neq 0$. Hence, we have $\llbracket \varphi \rrbracket_{\mathcal{M}, w, g[d / x]}^{\mathrm{s}} \neq 0$ and $\llbracket \psi \rrbracket_{\mathcal{M}, w, g[d / x]}^{\mathrm{s}} \neq 0$ for all $d \in \operatorname{Dom}(w)$. Hence, $\llbracket \varphi \wedge \psi \rrbracket_{\mathcal{M}, w, g}^{\mathrm{w}} \neq 0$ for all $d \in \operatorname{Dom}(w)$, contradicting (i).

Suppose (i) $\llbracket \forall x(\varphi \wedge \psi) \rrbracket_{\mathcal{M}, w, g}^{\mathrm{s}}=1$ but (ii) $\llbracket \forall x \varphi \wedge \forall x \psi \rrbracket_{\mathcal{M}, w, g}^{\mathrm{s}}=0$. By (ii), $\llbracket \forall x \varphi \rrbracket_{\mathcal{M}, w, g}^{\mathrm{w}}=0$ or $\llbracket \forall x \psi \rrbracket_{\mathcal{M}, w, g}^{\mathrm{w}}=0$. Hence, $\llbracket \varphi \rrbracket_{\mathcal{M}, w, g[d / x]}^{\mathrm{w}}=0$ for some $d \in \operatorname{Dom}(w)$ or $\llbracket \psi \rrbracket_{\mathcal{M}, w, g[d / x]}^{\mathrm{w}}=0$ for some $d \in \operatorname{Dom}(w)$. Hence, $\llbracket \varphi \wedge$ $\psi \rrbracket_{\mathcal{M}, w, g[d / x]}^{\mathrm{w}}=0$ for some $d \in \operatorname{Dom}(w)$, contradicting (i).

Suppose (i) $\llbracket \forall x(\varphi \wedge \psi) \rrbracket_{\mathcal{M}, w, g}^{\mathrm{s}}=1$ but (ii) $\llbracket \forall x \varphi \wedge \forall x \psi \rrbracket_{\mathcal{M}, w, g}^{\mathrm{s}}=\mathrm{u}$. By (ii), $\llbracket \forall x \varphi \rrbracket_{\mathcal{M}, w, g}^{\mathrm{S}}=\mathrm{u}$ or $\llbracket \forall x \psi \rrbracket_{\mathcal{M}, w, g}^{\mathrm{S}}=\mathrm{u}$. Hence, $\llbracket \varphi \rrbracket_{\mathcal{M}, w, g[d / x]}^{\mathrm{S}}=\mathrm{u}$ for some $d \in \operatorname{Dom}(w)$ or $\llbracket \psi \rrbracket_{\mathcal{M}, w, g[d / x]}^{\mathrm{s}}=\mathrm{u}$ for some $d \in \operatorname{Dom}(w)$. Hence, $\llbracket \varphi \wedge$ $\psi \rrbracket_{\mathcal{M}, w, g[d / x]}^{\mathrm{S}} \neq 1$ for some $d \in \operatorname{Dom}(w)$, contradicting (i).

Suppose (i) $\llbracket \forall x(\varphi \wedge \psi) \rrbracket_{\mathcal{M}, w, g}^{\mathrm{s}}=\mathrm{u}$ but (ii) $\llbracket \forall x \varphi \wedge \forall x \psi \rrbracket_{\mathcal{M}, w, g}^{\mathrm{s}}=0$. By (ii), $\llbracket \forall x \varphi \rrbracket_{\mathcal{M}, w, g}^{\mathrm{s}}=0$ or $\llbracket \forall x \psi \rrbracket_{\mathcal{M}, w, g}^{\mathrm{s}}=0$. Hence, $\llbracket \varphi \rrbracket_{\mathcal{M}, w, g[d / x]}^{\mathrm{s}}=0$ for some $d \in \operatorname{Dom}(w)$ or $\llbracket \psi \rrbracket_{\mathcal{M}, w, g[d / x]}^{\mathrm{s}}=0$ for some $d \in \operatorname{Dom}(w)$. Hence, $\llbracket \varphi \wedge$ $\psi \rrbracket_{\mathcal{M}, w, g[d / x]}^{\mathrm{S}}=0$ for some $d \in \operatorname{Dom}(w)$, contradicting (i).

Suppose (i) $\llbracket \forall x(\varphi \wedge \psi) \rrbracket_{\mathcal{M}, w, g}^{\mathrm{s}}=\mathrm{u}$ but (ii) $\llbracket \forall x \varphi \wedge \forall x \psi \rrbracket_{\mathcal{M}, w, g}^{\mathrm{s}}=1$. By (ii), $\llbracket \forall x \varphi \rrbracket_{\mathcal{M}, w, g}^{\mathrm{s}}=1$ and $\llbracket \forall x \psi \rrbracket_{\mathcal{M}, w, g}^{\mathrm{s}}=1$. Hence, we have $\llbracket \varphi \rrbracket_{\mathcal{M}, w, g[d / x]}^{\mathrm{s}}=1$ and $\llbracket \psi \rrbracket_{\mathcal{M}, w, g[d / x]}^{\mathrm{s}}=1$ for all $d \in \operatorname{Dom}(w)$. Hence, $\llbracket \varphi \wedge \psi \rrbracket_{\mathcal{M}, w, g[d / x]}^{\mathrm{s}}=1$ for all $d \in \operatorname{Dom}(w)$, contradicting (i).

As for principle 8, the proof is similar.

Theorem 2.1 now follows from Lemmas A.1, A.3, A.2 and A.4.

\section{A.2 Two Facts About Existence Claims}

Fact A.1 If $q \neq 0$ and $\mathrm{i} \in\{\mathrm{w}, \mathrm{s}\}$, then for any model $\mathcal{M}$, world $w$ and assignment $g, \llbracket \forall x \diamond \neg \exists y(y=x) \wedge \square \exists x(x=x) \rrbracket_{\mathcal{M}, w, g}^{i} \neq 1$. 
Proof Suppose $q \neq 0$ and $\mathrm{i} \in\{\mathrm{w}, \mathrm{s}\}$. Then

$\llbracket \forall x \diamond \neg \exists y(y=x) \rrbracket_{\mathcal{M}, w, g}^{\mathrm{i}}=1$ iff
$\llbracket \forall x \neg \square \neg \forall y(y \neq x) \rrbracket_{\mathcal{M}, w, g}^{\mathrm{i}}=1$ iff

for all $d \in \operatorname{Dom}(w), \llbracket \neg \square \neg \forall y(y \neq x) \rrbracket_{\mathcal{M}, w, g[d / x]}^{\mathrm{i}}=1$ iff

for all $d \in \operatorname{Dom}(w), \llbracket \square \neg \forall y(y \neq x) \rrbracket_{\mathcal{M}, w, g[d / x]}^{\mathrm{i}}=0$ only if

for all $d \in \operatorname{Dom}(w)$, there's $w^{\prime} \in W$ such that $w R w^{\prime}$ and

$\llbracket \neg \forall y(y \neq x) \rrbracket_{\mathcal{M}, w^{\prime}, g[d / x]}^{\mathrm{i}}=0$ iff

for all $d \in \operatorname{Dom}(w)$, there's $w^{\prime} \in W$ such that $w R w^{\prime}$ and

$\llbracket \forall y(y \neq x) \rrbracket_{\mathcal{M}, w^{\prime}, g[d / x]}^{\mathrm{i}}=1$ iff

for all $d \in \operatorname{Dom}(w)$, there's $w^{\prime} \in W$ such that $w R w^{\prime}$ and,

for all $d^{\prime} \in \operatorname{Dom}\left(w^{\prime}\right), \llbracket y \neq x \rrbracket_{\mathcal{M}, w^{\prime}, g[d / x]\left[d^{\prime} / y\right]}^{\mathrm{i}}=1$ iff

for all $d \in \operatorname{Dom}(w)$, there's $w^{\prime} \in W$ such that $w R w^{\prime}$ and,

for all $d^{\prime} \in \operatorname{Dom}\left(w^{\prime}\right), \llbracket y=x \rrbracket_{\mathcal{M}, w^{\prime}, g[d / x]\left[d^{\prime} / y\right]}^{\mathrm{i}}=0$ iff

for all $d \in \operatorname{Dom}(w)$, there's $w^{\prime} \in W$ such that $w R w^{\prime}$ and,

for all $d^{\prime} \in \operatorname{Dom}\left(w^{\prime}\right),\left\langle d, d^{\prime}\right\rangle \in \operatorname{Dom}\left(w^{\prime}\right)^{2}$ and $\left\langle d, d^{\prime}\right\rangle \notin I(=)\left(w^{\prime}\right)$ or $\left\langle d, d^{\prime}\right\rangle \notin$ $\operatorname{Dom}\left(w^{\prime}\right)^{2}$ and $\left\langle d, d^{\prime}\right\rangle \in I(=)\left(w^{\prime}\right)$,

which entails that $\operatorname{Dom}\left(w^{\prime}\right)=\emptyset$, in which case

$\llbracket \square \exists x(x=x) \rrbracket_{\mathcal{M}, w, g}^{\mathrm{i}} \neq 1$.

Fact A.2 If $q=0, p \neq 1$ and $\mathrm{i} \in\{\mathrm{w}, \mathrm{s}\}$, then for any model $\mathcal{M}$, world $w$ and assignment $g, \llbracket \exists x \diamond P x \wedge \square \forall x \neg P x \rrbracket_{\mathcal{M}, w, g}^{\mathrm{i}} \neq 1$.

Proof Suppose $q=0, p \neq 1$ and $\mathrm{i} \in\{\mathrm{w}, \mathrm{s}\}$. Then

$$
\begin{aligned}
& \llbracket \exists x \diamond P x \rrbracket_{\mathcal{M}, w, g}^{\mathrm{i}}=1 \text { iff } \\
& \llbracket \neg \forall x \neg \neg \square \neg P x \rrbracket_{\mathcal{M}, w, g}^{\mathrm{i}}=1 \text { iff } \\
& \llbracket \forall x \square \neg P x \rrbracket_{\mathcal{M}, w, g}^{\mathrm{i}}=0, \text { only if }
\end{aligned}
$$

there's $d \in \operatorname{Dom}(w)$ s.t. $\llbracket \square \neg P x \rrbracket_{\mathcal{M}, w, g[d / x]}^{\mathrm{i}}=0$, only if

there's $d \in \operatorname{Dom}(w)$ and $w^{\prime} \in W$ s.t. $w R w^{\prime}$ and

$\llbracket \neg P x \rrbracket_{\mathcal{M}, w^{\prime}, g[d / x]}^{i}=0$, iff

there's $d \in \operatorname{Dom}(w)$ and $w^{\prime} \in W$ s.t. $w R w^{\prime}$ and

$\llbracket P x \rrbracket_{\mathcal{M}, w^{\prime}, g[d / x]}^{\mathrm{i}}=1$, iff

there's $d \in \operatorname{Dom}(w)$ and $w^{\prime} \in W$ s.t. $w R w^{\prime}$ and

$d \in \operatorname{Dom}\left(w^{\prime}\right)$ and $d \in I(P)\left(w^{\prime}\right)$, in which case

$\llbracket \square \forall x \neg P x \rrbracket_{\mathcal{M}, w, g}^{\mathrm{i}} \neq 1$.

\section{References}

1. Fitting, M., \& Mendelsohn, R.L. (1998). First-order modal logic. Dordrecht: Kluwer Academic.

2. Gamut, L.T.F. (1991). Logic, language, and meaning. Vol. 2, Intensional logic and logical grammar. Chicago: University of Chicago Press.

3. Kripke, S.A. (1963). Semantical considerations on modal logic. Acta Philosophica Fennica, 16(1963), 83-94.

4. Nolt, J. (2014). Free logic. In Zalta, E.N. (Ed.) The Stanford Encyclopedia of Philosophy. Metaphysics Research Lab, Stanford University, winter 2014 edition. 